\title{
Klinotaxis as a basic form of navigation
}

\section{Dominique Martinez *}

UMR 7503, Laboratoire Lorrain de Recherche en Informatique et ses Applications, Centre National de la Recherche Scientifique, Vandoeuvre-lès-Nancy, France *Correspondence: dominique.martinez@loria.fr

Edited by:

Martin Giurfa, Université Paul Sabatier-Toulouse III, France

Reviewed by:

Matthieu Louis, CRG, Spain

Simon Benhamou, CNRS, France

Keywords: klinotaxis, weathervaning, proportional navigation, Drosophila larva, C. elegans

\section{A commentary on}

Multilevel control of run orientation in Drosophila larval chemotaxis

by Gomez-Marin A., and Louis $M$. (2014). Front. Behav. Neurosci. 8:38. doi: 10.3389/fnbeh.2014.00038

In their article, Gomez-Marin and Louis (2014) found that runs in Drosophila larval chemotaxis bend toward the direction of higher concentration. This steering process called weathervaning or klinotaxis was previously discovered in the worm C. elegans (Ward, 1973; Iino and Yoshida, 2009) and raises the question: how is it performed? Although Gomez-Marin and Louis (2014) found that run orientation relies on the detection of the lateral gradient component, the underlying control mechanism is largely unknown. Two alternative strategies might be employed, direct error correction and proportional navigation (Figure 1A). In direct error correction, the animal would turn proportionally to the error; that is, the local bearing angle between the current heading and the desired direction to the goal (intensity peak). In proportional navigation (Murtaugh and Criel, 1966; Zarchan, 2012), the objective is to maintain a constant line of sight (LOS) angle during motion (Figure 1A). This tactic has been known by sailors for many years as a mean to detect collision courses and is currently implemented in guided missiles. Thus, in proportional navigation, the animal would turn proportionally to the derivative of the LOS angle. Here we argue that klinotaxis, as observed in Gomez-Marin and Louis (2014), does not result from direct error correction. Instead, klinotaxis is directed through proportional navigation that is a basic form of navigation (where the goal direction is estimated using gradient sensing rather than spatial memory) involving indirect error correction through an attempt to keep the LOS angle constant. Our claim is supported by theoretical analyses as well as computer simulations.

\section{WHAT KIND OF MOTOR OUTPUT RESULTS FROM DIRECT ERROR CORRECTION AND PROPORTIONAL NAVIGATION?}

The agent, either Drosophila larva or C. elegans, is modeled as a single point $(\mathrm{x}, \mathrm{y})$ moving at constant speed $\mathrm{v}$. The equations of motion are given by

$$
\begin{aligned}
& \frac{d x}{d t}=v \cos \gamma \\
& \frac{d y}{d t}=v \sin \gamma
\end{aligned}
$$

where $\gamma$ is the direction of movement with respect to a global reference (Figure 1A). The agent aims at orienting toward the goal by controlling its turning rate $\omega=$ $d \gamma / d t$. The orientation error is given by the local bearing angle $\beta=\alpha-\gamma$ where $\alpha$ is the line of sight (LOS) angle; that is, the absolute direction of the beeline from the agent to the goal. What kind of motor output may result from direct error correction and proportional navigation?

A direct error correction strategy would produce a motor output (turning rate $\omega$ ) proportional to the error (local bearing angle $\beta$ )

$$
\omega=K \beta
$$

with proportional constant K. Yet, no evidence of such a linear relationship was found in (Gomez-Marin and Louis, 2014). Instead, Figure 1B clearly shows that $\omega \simeq K \sin \beta$. A similar turning rate function is observed in $C$. elegans klinotaxis (see Figure 2A in Iino and Yoshida, 2009) and in other types of taxis, e.g., gyrotaxis (Codling et al., 2008). Can such sinusoidal reorientations be obtained with proportional navigation? Proportional navigation is a dedicated term for a basic form of navigation that aims at maintaining the LOS angle $\alpha$ constant during motion (Murtaugh and Criel, 1966; Zarchan, 2012). It was originally developed for missile guidance toward moving targets. Recently, it has been employed for navigating a robot toward a fixed goal with the kinematics given by Equation (1) and was defined as follows (Belkhouche and Belkhouche, 2007).

$$
\omega=K \frac{d \alpha}{d t}
$$

so that the agent moves in a straight line when $\alpha$ remains constant. In the Supplementary Materials, we show that the rate of change of $\alpha$ is given by $v \sin \beta / r$ so that

$$
\omega=K \frac{v}{r} \sin \beta
$$

Thus, proportional navigation is expected to produce a motor output $\omega$ proportional to the sine of $\beta$, similar to that observed experimentally (Figure 1B). Note however that Equation (4) is useless in practice as both the distance $r$ to the goal and local bearing angle $\beta$ are unknown to the agent. 
A Error correction vs proportional navigation

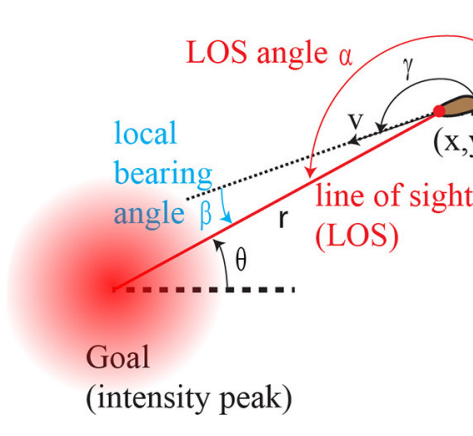

\section{Longitudinal sampling}

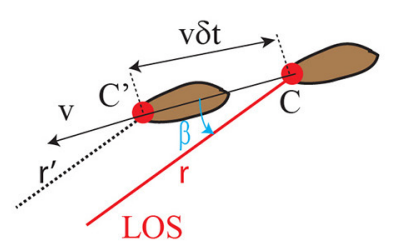

Turning rate:

$$
\omega=\frac{\mathrm{d} \gamma}{\mathrm{dt}}
$$

Error correction:

$$
\omega=\mathrm{K} \beta
$$

Proportional navigation:

$$
\omega=K \frac{\mathrm{d} \alpha}{\mathrm{dt}}
$$

D Lateral sampling

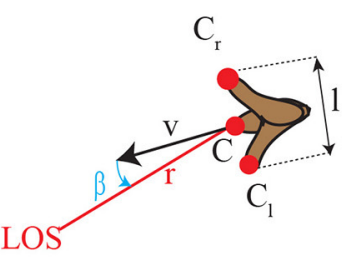

B Modulation of the turning rate

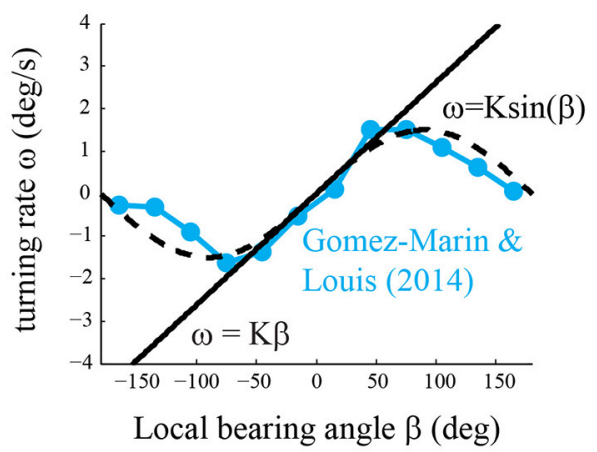

E Simulated proportional navigation

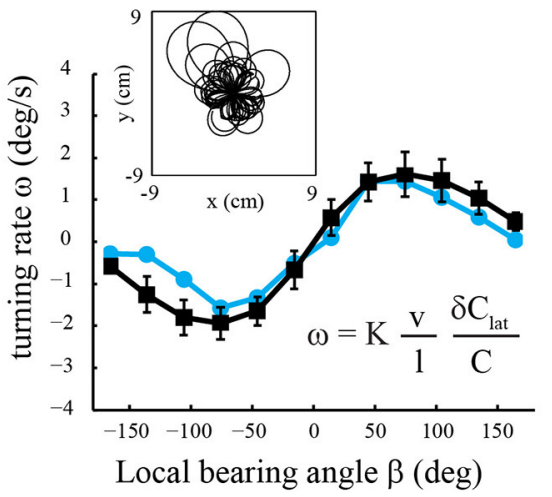

FIGURE 1 | (A) Error correction vs. proportional navigation. The turning rate is proportional to the local bearing angle in error correction and to the rate of change of the LOS angle in proportional navigation. See text for the details. (B) Dependency of the turning rate on the local bearing angle. The experimental data (in blue) are reprinted with modification from Figure 1C in Gomez-Marin and Louis (2014). The straight line represents error correction (Equation 2 with $K=1.5$ ). The dashed curve is for $\omega=K \sin \beta$ with $K=1.5$. (C) Longitudinal sampling of the gradient along the direction of motion while the animal moves forward. See text for the details. (D) Lateral sampling of the gradient perpendicular to the direction of motion during head sweeps. See text for the details. (E) Dependency of the turning rate on the local bearing angle for simulated proportional navigation (black curve, mean \pm s.d., $n=100$ trajectories) as compared to experimental data (blue curve, same as in panel B). The inset shows a superposition of the trajectories obtained with proportional navigation. Simulation details are as follows: the steady-state concentration field for a source that emits continuously with rate $F=0.1 \mathrm{mM} / \mathrm{s}$ and diffusivity $D=0.05 \mathrm{~cm}^{2} / \mathrm{s}$ is given by $C(r)=F /(4 \pi D r)$. The agent starts at a random location $\left(x_{0}, y_{0}\right)$ at $r(t=0)=3 \mathrm{~cm}$ from the odor source. Its initial orientation is taken randomly between $-\pi$ and $\pi$. The equations of motion (Equation 1 as well as $\omega=d \gamma / d t$ ) are integrated with a time step of $0.1 \mathrm{~s}$. The speed of the agent is $v=0.4 \mathrm{~mm} / \mathrm{s}$. The turning rate $\omega$ is calculated from Equation (7) with $K=3, I=1 \mathrm{~mm}, \delta C_{\text {lat }}=C_{l}-C_{r}$ (see panel D) and $C=\left(C_{l}+C_{r}\right) / 2$. To account for intermittent and noisy observations, the relative concentration change $\left(\delta C_{\text {lat }} / C\right)$ is not sampled at every time step but with 0.1 probability and multiplicative noise $\eta\left(\delta C_{\text {lat }} / C\right)$ is added to the measurements $(\eta=$ uniform random variable with mean $=0$ and s.d. $=0.1$ ).
The sensory input required for proportional navigation is examined in the next section.

\section{WHAT KIND OF SENSORY INFORMATION IS NEEDED FOR DIRECT ERROR CORRECTION AND PROPORTIONAL NAVIGATION?}

Drosophila larva and C. elegans assess the chemical gradient by comparing the stimulus intensity over time. Yet, it is not known exactly how the gradient is sampled. It could be sampled along the direction of motion while the animal moves forward (Ferrée and Lockery, 1999) or perpendicular to the direction of motion during head sweeps (Ward, 1973; Iino and Yoshida, 2009; Gomez-Marin and Louis, 2014). The Drosophila larva moves at $v \simeq 0.5-1 \mathrm{~mm} / \mathrm{s}$ and may integrate information on time scales of a few seconds, and low-amplitude head sweeps lead to side-by-side deflections of the head of $l \simeq 0.7 \mathrm{~mm}$ (Gomez-Marin and Louis, 2014). Thus, measurements along or perpendicular to the direction of motion would be performed over distances of about $1 \mathrm{~mm}$. Applying the same logic as in Gomez-Marin et al. (2010), we estimate that concentration differences are 10-fold larger than the noise level so that both lateral and longitudinal samplings are viable options. In what follows, we therefore investigate the two possibilities.

In Figure 1C, we consider that the agent takes measurements every $\delta t$ sec during forward locomotion. The longitudinal gradient component is estimated along the direction of motion as $\delta C_{\text {lon }} /(v \delta t)$ where $\delta C_{\text {lon }}$ is the measured difference in concentration and $v \delta t$ is the distance traveled 
between two consecutive measurements. In the Supplementary Materials, we show that the longitudinal gradient component is related to the local bearing angle as follows.

$$
\cos \beta \simeq \frac{r}{C}\left(\frac{\delta C_{l o n}}{v \delta t}\right)
$$

In Figure 1D, we consider side-to-side movements of the head sensor of amplitude $l$ perpendicular to the direction of motion so that the lateral gradient component is estimated as $\delta C_{\text {lat }} / l$ where $\delta C_{\text {lat }}$ is the difference in concentration from one side to the other. In the Supplementary Materials, we show that the lateral gradient component is related to the local bearing angle as follows.

$$
\sin \beta \simeq \frac{r}{C}\left(\frac{\delta C_{l a t}}{l}\right)
$$

How to make use of this sensory information? From Equation (2), error correction requires the value of $\beta$. Taking the inverse cosine of Equation (5) leads to $\pm \beta$ whereas taking the inverse sine of Equation (6) leads to $\beta$ or $\pi-\beta$. These ambiguities prevent the use of either the lateral or the longitudinal gradient component alone. In contrast, an efficient implementation of Equation (2) using both longitudinal and lateral gradient components is provided by $\omega=K$ atan $2(\sin \beta, \cos \beta)$ where atan 2 is the four-quadrant inverse tangent.

From Equation (4), proportional navigation requires the value of $\sin \beta$. On the one hand, the use of Equation (5) leads to $\sin \beta= \pm \sqrt{1-\cos ^{2} \beta}$ which makes the turning direction unknown. On the other hand, the lateral gradient component alone is sufficient for proportional navigation. From Equations (4) and (6), proportional navigation writes as follows.

$$
\omega=K \frac{v}{l}\left(\frac{\delta C_{l a t}}{C}\right)
$$

We note that the turning rate $\omega=$ $d \gamma / d t$ in Equation (7) is proportional to the relative change in stimulus intensity $\delta C_{\text {lat }} / C$ so that proportional navigation obeys the Weber-Fechner law (Kandel et al., 2000). Figure 1E shows numerical results of Equation (7) (simulation details in Figure caption) that are in good agreement with experimental data. We also note that the robotic implementation of Equation (7) appeared to be very robust in real conditions (see illustrative video at http://youtu.be/XvVERq4 h_uc).

From experimental studies (Iino and Yoshida, 2009; Gomez-Marin and Louis, 2014), we know that the relationship between turning rate and bearing error in Drosophila larva and C. elegans is approximately sinusoidal. Also, in both animals, the turning rate is clearly correlated with the lateral gradient component and not with the longitudinal gradient component. Here, we show that proportional navigation produces a sinusoidal relationship between turning rate and bearing error and merely employs the lateral gradient component. In contrast, direct error correction is linear per $s e$ and requires both lateral and longitudinal gradient components. Together, these data suggest that klinotaxis reflects proportional navigation rather than direct error correction. Nevertheless, because it is fairly impossible to rule out that another model may explain the data equally well, the conclusion that klinotaxis is directed through proportional navigation remains a viable hypothesis in the absence of compelling experimental evidence.

Proportional navigation is a strategy employed by certain predators to track unpredictably moving targets, e.g., bats chasing insects (Ghose et al., 2006). In a prey pursuit, proportional navigation results in an advantage for the pursuer that is known as motion camouflage (Mizutani et al., 2003). What could be the interest of proportional navigation for klinotaxis? At first sight, the sine relationship between turning rate and bearing error does not seem optimal because small turning rates are produced for large bearing errors. However, the continuity of the sinus with zero crossing at $\pm 180^{\circ}$ may prevent chaotic reorientations in the presence of noise. In addition, proportional navigation (unlike direct error correction) requires minimal sensory information; i.e., the lateral gradient component. Thus, Drosophila larvae and C. elegans may prefer proportional navigation over direct error correction because of its simplicity and robustness, just like proportional navigation is the preferred method of missile guidance because it provides the best performance with minimal sensory information (Murtaugh and Criel, 1966; Zarchan, 2012).

\section{ACKNOWLEDGMENT}

This work was funded by the state program investissements d'avenir managed by ANR (grant ANR-10-BINF-05 "Pherotaxis").

\section{SUPPLEMENTARY MATERIAL}

The Supplementary Material for this article can be found online at: http://www. frontiersin.org/journal/10.3389/fnbeh.2014. 00275/full

\section{REFERENCES}

Belkhouche, F., and Belkhouche, B. (2007). Wheeled mobile robot navigation using proportional navigation. Adv. Robot. 21, 395-420. doi: $10.1163 / 156855307780132036$

Codling, E. A., Plank, M. J., and Benhamou, S. (2008). Random walk models in biology. J. R. Soc. Interface 5, 813-834. doi: 10.1098/rsif. 2008.0014

Ferrée, T. C., and Lockery, S. R. (1999). Computational rules for chemotaxis in the nematode C. elegans. J. Comput. Neurosci. 6, 263-277.

Ghose, K., Horiuchi, T. K., Krishnaprasad, P. S., and Moss, C. F. (2006). Echolocating bats use a nearly time-optimal strategy to intercept prey. PLoS Biol. 4:e108. doi: 10.1371/journal.pbio. 0040108

Gomez-Marin, A., Duistermars, B. J., Frye, M. A., and Louis, M. (2010). Mechanisms of odortracking: multiple sensors for enhanced perception and behavior. Front. Cell. Neurosci. 4:6. doi: 10.3389/fncel.2010.00006

Gomez-Marin, A., and Louis, M. (2014). Multilevel control of run orientation in Drosophila larval chemotaxis. Front. Behav. Neurosci. 8:38. doi: 10.3389/fnbeh.2014.00038

Iino, Y., and Yoshida, K. (2009). Parallel use of two behavioral mechanisms for chemotaxis in Caenorhabditis elegans. J. Neurosci. 29, 5370-5380. doi: 10.1523/JNEUROSCI.363308.2009

Kandel, E. R., Schwartz, J. H., and Jessell, T. M. (2000). Principles of Neural Science, 4th Edn. New York, NY: McGraw-Hill.

Mizutani, A., Chahl, J. S., and Srinivasan, M. V. (2003). Insect behaviour: motion camouflage in dragonflies. Nature 423:604. doi: 10.1038/423604a

Murtaugh, S. A., and Criel, H. E. (1966). Fundamentals of proportional navigation. IEEE Spectr. 3, 75-85. 
Ward, S. (1973). Chemotaxis by the nematode Caenorhabditis elegans: identification of attractants and analysis of the response by use of mutants. Proc. Natl. Acad. Sci. U.S.A. 70, 817-821. doi: 10.1073/pnas.70.3.817

Zarchan, P. (2012). Tactical and Strategic Missile Guidance, 6th Edn. Reston: AIAA publisher.

Conflict of Interest Statement: The author declares that the research was conducted in the absence of any commercial or financial relationships that could be construed as a potential conflict of interest.

Received: 23 May 2014; accepted: 24 July 2014; published online: 14 August 2014.

Citation: Martinez D (2014) Klinotaxis as a basic form of navigation. Front. Behav. Neurosci. 8:275. doi: 10.3389/fnbeh.2014.00275

This article was submitted to the journal Frontiers in Behavioral Neuroscience.
Copyright (C) 2014 Martinez. This is an open-access article distributed under the terms of the Creative Commons Attribution License (CC BY). The use, distribution or reproduction in other forums is permitted, provided the original author(s) or licensor are credited and that the original publication in this journal is cited, in accordance with accepted academic practice. No use, distribution or reproduction is permitted which does not comply with these terms. 